\title{
The distance to M31 in the era of precision cosmology
}

\section{David Valls-Gabaud}

LERMA, UMR CNRS 8112, Observatoire de Paris, 61 Avenue de l'Observatoire, 75014 Paris, France

email: david.valls-gabaud@obspm.fr

\begin{abstract}
With the advent of precision cosmology, where distances out to redshifts $z<0.6$ can be measured to $2 \%$ precision on the basis of baryon acoustic oscillations, it appears essential to establish an accurate calibration of the primary and secondary indicators of the cosmological distance ladder. Here we review recent attempts at anchoring M31 very accurately using three independent methods, and discuss in detail the systematics that affect each. Two double-lined eclipsing binaries yield a distance to M31 which is precise to 4\%. New Bayesian methods have been applied to determine the tip of the red-giant branch, even in sparsely populated colourmagnitude diagrams, and provide unique insights in the context of a precise three-dimensional distribution of the satellites in the M31 system. Over 2500 Cepheids have been identified in largescale multi-colour surveys of M31, the largest homogeneous data set thus far obtained for any galaxy. A subset of 68 with periods longer than 10 days have been observed with the Wide-Field Camera 3 on board the Hubble Space Telescope, yielding the tightest-ever near-infrared periodluminosity relation, with a mean distance error of $1 \%$. Combined with other measurements, the distance to M31 is now measured with a precision of $3 \%$. Forthcoming improvements, and their implications, are also discussed.
\end{abstract}

Keywords. binaries: eclipsing, stars: distances, Cepheids, galaxies: individual (M31), Local Group 